\title{
Astérisque
}

\section{JEAN-MARC DESHOUILLERS}

GREGORY A. FREIMAN

\section{On an additive problem of Erdôs and Straus, 2}

Astérisque, tome 258 (1999), p. 141-148

<http://www.numdam.org/item?id=AST_1999_258_141_0>

(C) Société mathématique de France, 1999, tous droits réservés.

L'accès aux archives de la collection « Astérisque » (http://smf4.emath.fr/ Publications/Asterisque/) implique l'accord avec les conditions générales d'utilisation (http://www.numdam.org/conditions). Toute utilisation commerciale ou impression systématique est constitutive d'une infraction pénale. Toute copie ou impression de ce fichier doit contenir la présente mention de copyright.

\section{Numdam}


Astérisque 258, 1999, p. 141-148

\title{
ON AN ADDITIVE PROBLEM OF ERDÕS AND STRAUS, 2
}

\author{
by \\ Jean-Marc Deshouillers \& Gregory A. Freiman
}

\begin{abstract}
We denote by $s^{\wedge} A$ the set of integers which can be written as a sum of $s$ pairwise distinct elements from $A$. The set $A$ is called admissible if and only if $s \neq t$ implies that $s^{\wedge} A$ and $t^{\wedge} A$ have no element in common.

P. Erdôs conjectured that an admissible set included in $[1, N]$ has a maximal cardinality when $A$ consists of consecutive integers located at the upper end of the interval $[1, N]$. The object of this paper is to give a proof of Erdôs' conjecture, for sufficiently large $N$.
\end{abstract}

Let $\mathcal{A}$ be a set of positive integers having the property that each time an integer $n$ can be written as a sum of distinct elements of $\mathcal{A}$, the number of summands is well defined, in that the integer $n$ cannot be written as a sum of distinct elements of $\mathcal{A}$ with a different number of summands. This notion has been introduced by P. Erdôs in 1962 (cf. [2]) and called admissibility by E.G. Straus in 1966 (cf. [5]). In other words, if we denote by $s^{\wedge} \mathcal{A}$ the set of integers which can be written as a sum of $s$ pairwise distinct elements from $\mathcal{A}$ then $\mathcal{A}$ is admissible if and only if $s \neq t$ implies that $s^{\wedge} \mathcal{A}$ and $t^{\wedge} \mathcal{A}$ have no element in common.

Erdôs conjectured that an admissible subset $\mathcal{A}$ included in $[1, N]$ has a cardinality which is maximal when $\mathcal{A}$ consists of consecutive integers located at the upper end of the interval $[1, N]$. As it was computed by E.G. Straus, the set

$$
\{N-k+1, N-k+2, \ldots, N\}
$$

is admissible if and only if $k \leq 2 \sqrt{N+1 / 4}-1$.

Straus himself proved that $\sqrt{N}$ is the right order of magnitude for the cardinality of a maximal admissible subset from $[1, N]$. More precisely, he proved the inequality $|\mathcal{A}| \leq(4 / \sqrt{3}+o(1)) \sqrt{N}$. The constant involved has been slightly reduced by $\mathrm{P}$. Erdôs, J-L. Nicolas and A. Sárkốzy (cf. [3]) and we proved (cf. [1] the inequality

1991 Mathematics Subject Classification. - 11 P99, 05 D05.

Key words and phrases. - Admissible sets, arithmetic progressions.

J.-M. D.: Cette recherche a bénéficié du soutien du CNRS (UMR 9936, Université Bordeaux 1) et de l'Université Victor Segalen Bordeaux 2. 
$|\mathcal{A}| \leq(2+o(1)) \sqrt{N}$. The object of this paper is to give a proof of Erdôs conjecture, at least when $N$ is sufficiently large.

Theorem 1. - There exists an integer $N_{0}$, effectively computable, such that for any integer $N \geq N_{0}$ and any admissible subset $\mathcal{A} \subset[1, N]$ we have

$$
\text { Card } \mathcal{A} \leq 2 \sqrt{N+1 / 4}-1 \text {. }
$$

The proof is based on the description of the structure of large admissible sets we obtained previously, namely :

Theorem 2 (J-M. Deshouillers, G.A. Freiman [1]). - Let $\mathcal{A}$ be an admissible set included in $[1, N]$, such that $\operatorname{Card} \mathcal{A}>1.96 \sqrt{N}$. If $N$ is large enough, there exist $\mathcal{C} \subset \mathcal{A}$ and an integer $q$ having the following properties :

(i) $\operatorname{Card} \mathcal{C} \leq 10^{5} N^{5 / 12}$,

(ii) for some $t$ the set $t^{\wedge} \mathcal{C}$ contains at least $3 N^{5 / 6}$ terms in an arithmetic progression modulo $q$,

(iii) $\mathcal{A} \backslash \mathcal{C}$ is included in an arithmetic progression modulo $q$ containing at most $N^{7 / 12}$ terms.

Although we do not develop this point, it will be clear from the proof that our arguments may be used to describe the structure of maximal admissible subsets of $[1, N]$, leading for example to the fact that when $N$ has the shape $n^{2}$ or $n^{2}+n$ (and $n$ sufficiently large), the Erdôs - Straus example is the only maximal subset of $[1, N]$.

1. We first establish a lemma expressing the fact that if a set of integers $\mathcal{D}$ is part of a finite arithmetic progression with few missing elements, then the same is locally true for $s^{\wedge} \mathcal{D}$.

Proposition 1. - Let us consider integers $r, s, t$ and $a, q$ such that $t \geq 2 s-q, \quad s \geq$ $4 r+3+q$ and $0 \leq a<q$.

Let further $\mathcal{D}=\left\{d_{1}<d_{2}<\cdots<d_{t}\right\}$ be a set of $t$ distinct integers congruent to a modulo $q$ such that $d_{t}-d_{1}=(t-1+r) q$, and denote by $m$ (resp. $\left.M\right)$ the smallest (resp. largest) element in $s^{\wedge} \mathcal{D}$. Then, among $2 r+1$ consecutive integers congruent to sa modulo $q$ and laying in the interval $[m, M]$, at least $r+1$ belong to $s^{\wedge} \mathcal{D}$.

Proof. - We treat the special case when $a=0, q=1$ and $\mathcal{D}$ is included in $[1, t]$. We notice that the general case reduces to this one by writing $d_{l}=d_{1}+q\left(\delta_{l}-1\right)$ and considering the set $\left\{\delta_{1}, \ldots, \delta_{t}\right\}$.

Let $x$ be an integer in $s^{\wedge} \mathcal{D} \cap[m,(m+M) / 2]$. We first show that the interval $[x, x+3 r]$ contains at least $2 r+1$ elements from $s^{\wedge} \mathcal{D}$. Since $x$ is in $s^{\wedge} \mathcal{D}$, we can find $d(1)<\cdots<d(s)$, elements in $\mathcal{D}$, the sum of which is $x$.

Let us show that $d(1)$ is less than $t-s-3 r$. On the one hand we have

$$
m+M \leq(r+1)+\cdots+(r+s)+(t+r-s+1)+\cdots+(t+r)=\frac{s}{2}(2 t+4 r+2)
$$

and on the other hand we have

$$
x \geq d(1)+(d(1)+1)+\cdots+(d(1)+s-1)=\frac{s}{2}(2 d(1)+s-1) .
$$


The inequality $x \leq(m+M) / 2$ implies that we have

$$
2 d(1)+s-1 \leq t+2 r+1,
$$

whence

$$
2 d(1) \leq 2(t-s-3 r)-(t-s-4 r-2),
$$

and we notice that $t-s-4 r-2$ is positive, by the assumptions of Proposition 1 .

Since $d(1)$ is less than $t-s-3 r$, the interval $[d(1), t+r]$ contains at least $s+4 r+1$ integers. We denote by $i_{1}<\cdots<i_{l}$ the indexes of those $d^{\prime} s$ such that $d\left(i_{k}+1\right)-$ $d\left(i_{k}\right) \geq 2$, with the convention that $d\left(i_{l}+1\right)=3 D t+r+1$ in the case when $d(s)<t+r$. The set

$$
\left.\bigcup_{k=1}^{l}\right] d\left(i_{k}\right)+1, d\left(i_{k}+1\right)-1[
$$

contains at least $4 r+1$ integers. We now suppress from those intervals those which contain no element from $\mathcal{D}$, and we rewrite the remaining ones as

$$
] d\left(j_{1}\right)+1, d\left(j_{1}+1\right)-1[, \ldots,] d\left(j_{h}\right)+1, d\left(j_{h}+1\right)-1[\text {. }
$$

They contain at least $3 r+1$ integers, among which at most $r$ are not in $\mathcal{D}$.

Let us define $u_{1}$ to be the largest integer such that $d\left(j_{1}\right)+u_{1}$ is in $\mathcal{D}$ and is less than $d\left(j_{1}+1\right)$, and let us define $u_{2}, \ldots, u_{h}$ in a similar way. We consider the integers

$$
\begin{gathered}
x=y+d\left(j_{1}\right)+\cdots+d\left(j_{h}\right) \quad(\text { which defines } y), \\
x+1=y+d\left(j_{1}\right)+1+d\left(j_{2}\right)+\cdots+d\left(j_{h}\right), \\
\cdots \\
x+u_{1}=y+d\left(j_{1}\right)+u_{1}+d\left(j_{2}\right)+\cdots+d\left(j_{h}\right), \\
\cdots \\
x+u_{1}+\cdots+u_{h}=y+d\left(j_{1}\right)+u_{1}+d\left(j_{2}\right)+u_{2}+\cdots+d\left(j_{h}\right)+u_{h} .
\end{gathered}
$$

One readily deduces from this construction that the interval

$$
\left[x, x+\min \left(3 r, u_{1}+\cdots+u_{h}\right)\right]
$$

contains at most $r$ elements which are not in $s^{\wedge} \mathcal{D}$.

What we have proven so far easily implies that any interval $[z-r, z]$ with $m \leq$ $z \leq(M+m) / 2$ contains at least one element in $s^{\wedge} \mathcal{D}$. Let us consider an interval $[y, y+2 r]$ with $m \leq y \leq(M+m) / 2$. By what we have just said, the interval $[y-r, y]$ contains an element in $s^{\wedge} \mathcal{D}$, let us call it $x$. As we have shown the interval $[x, x+3 r]$ contains at most $r$ integers not in $s^{\wedge} \mathcal{D}$, so that $[y, y+2 r]$ contains at most $r$ integers not in $s^{\wedge} \mathcal{D}$, which is equivalent to say that it contains at least $r+1$ elements from $s^{\wedge} \mathcal{D}$.

A similar argument taking into account decreasing sequences and starting with $M$ shows that any interval $[y-2 r, y]$ with $(m+M) / 2 \leq y \leq M$ contains at least $r+1$ elements from $s^{\wedge} \mathcal{D}$. 
2. We now prove the following result concerning the structure of a large admissible finite set.

Theorem 3. - Let $\mathcal{A}=\left\{a_{1}<\cdots<a_{A}\right\}$ be an admissible subset of $[1, N]$ with cardinality $A=2 N^{1 / 2}+O\left(N^{5 / 12}\right)$, and let us define $q$ to be the largest integer such that $\mathcal{A}$ is contained in an arithmetic progression modulo $q$. We have $q=O\left(N^{5 / 12}\right)$ and there exists an integer $u$ in $\left[N^{11 / 24}, 2 N^{11 / 24}\right]$ such that

$$
a_{A-u}-a_{u+1}=q\left(2 N^{1 / 2}+O\left(N^{11 / 24}\right)\right) .
$$

Proof. - The proof is based on the structure result we quoted in the introduction as Theorem 2. We keep its notation and first show that an integer $q$ satisfying (ii) and (iii) is indeed the largest integer such that $\mathcal{A}$ is contained in an arithmetic progression modulo $q$. We let $\mathcal{B}$ denote $\mathcal{A} \backslash \mathcal{C}$.

A simple counting argument will show that $\mathcal{A}$ is included in the same arithmetic progression as $\mathcal{B}$. Otherwise, let us consider an element $a \in \mathcal{A}$ which is not in the same arithmetic progression as $\mathcal{B}$ modulo $q$. The set $s^{\wedge} \mathcal{A}$ contains the disjoint sets $s^{\wedge} \mathcal{B}$ and $a+(s-1)^{\wedge} \mathcal{B}$. We thus have $\left|s^{\wedge} \mathcal{A}\right| \geq\left|s^{\wedge} \mathcal{B}\right|+\left|(s-1)^{\wedge} \mathcal{B}\right|$. It is well-known (cf. [4] for example) that $\left|s^{\wedge} \mathcal{B}\right| \geq s(|\mathcal{B}|-s)$ for $s \leq|\mathcal{B}|$, and since $\mathcal{A} \subset[1, N]$ is admissible we have

$$
\begin{aligned}
N(|\mathcal{B}|+1) & \geq \quad \operatorname{Card}\left(\bigcup_{s}\left(s^{\wedge} \mathcal{B} \cup\left(a+(s-1)^{\wedge} \mathcal{B}\right)\right)\right. \\
& \geq 2 \sum_{s}\left|s^{\wedge} \mathcal{B}\right| \geq 2 \sum_{s} s=20(|\mathcal{B}|-s)=\frac{1}{3}|\mathcal{B}|^{3}+O(N),
\end{aligned}
$$

which implies $|\mathcal{B}| \leq(\sqrt{3}+o(1)) \sqrt{N}$, so that we have $|\mathcal{A}|=|\mathcal{B}|+|\mathcal{C}| \leq(\sqrt{3}+o(1)) \sqrt{N}$ a contradiction.

We have so far proven that $q$ divides $g:=\operatorname{gcd}\left(a_{2}-a_{1}, \ldots, a_{A}-a_{1}\right)$. Property (ii) implies that $q$ is a multiple of $g$, so that we have $q=g$, as we wished to show.

The second step in the proof consists in showing that for $0<k \leq|\mathcal{B}|-q$, any element in $k^{\wedge} \mathcal{B}$ is less than any element in $(k+q)^{\wedge} \mathcal{B}$. Let us call $J$ the $3 N^{5 / 6}$ consecutive terms of the arithmetic progression modulo $q$, the existence of which is asserted in (ii). Since $\mathcal{B}$ is included in an arithmetic progression modulo $q$ with less that $3 N^{5 / 6}$ terms, the sets $k^{\wedge} \mathcal{B}+J$ and $(k+q)^{\wedge} \mathcal{B}+J$ consists of consecutive terms of arithmetic progressions modulo $q$, and moreover, they are in the same class modulo $q$. Since $\mathcal{A}$ is admissible, the sets $k^{\wedge} \mathcal{B}+J$ (included in $\left.(k+t)^{\wedge} \mathcal{A}\right)$ and $(k+q)^{\wedge} \mathcal{B}+J$ (included in $\left.(k+q+t)^{\wedge} \mathcal{A}\right)$ do not intersect. To prove that any element of $k^{\wedge} \mathcal{B}$ is less that any element of $(k+q)^{\wedge} \mathcal{B}$, it is now sufficient to notice that $k^{\wedge} \mathcal{B}$ contains an element (we can consider the smallest element of $k^{\wedge} \mathcal{B}$ ), which is smaller than some element of $(k+q)^{\wedge} \mathcal{B}$.

We now prove that $q=O\left(N^{5 / 12}\right)$. The cardinality of $\mathcal{A}$ and Theorem 2 imply that $|\mathcal{B}|=2 N^{1 / 2}+O\left(N^{5 / 12}\right)$. We choose $k$ so that $2 k+q$ is $|\mathcal{B}|$ or $|\mathcal{B}|-1$. (We notice that this is always possible since $\mathcal{A}$ contains at least $N^{1 / 2}$ integers from $[1, N]$ in an arithmetic progression modulo $q$, so that $q \leq N^{1 / 2}$ ). By the second step, the largest element in $k^{\wedge} \mathcal{B}$ is smaller than the largest element in $(k+q)^{\wedge} \mathcal{B}$. Let $z$ be $(k+q)$-th element from $\mathcal{B}$, in the increasing order. We have

$$
z \leq N-(k-1) q
$$


and

$$
(z+q)+\cdots+(z+q k) \leq z+(z-q)+\cdots+(z-(k+q-1) q)
$$

by an easy computation, we get

$$
(q+2 k)^{2} \leq 2 N+2 k^{2}+3 q,
$$

but $2 k+q=|\mathcal{B}|+O(1)=|\mathcal{A}|+O\left(N^{5 / 12}\right)$, which implies

$$
2 k^{2} \geq 2 N\left(1+O\left(N^{-1 / 12}\right)\right)
$$

so that we have

$$
k=N^{1 / 2}+O\left(N^{5 / 12}\right) .
$$

We now use again the same argument, being more precise. Let us write $\mathcal{B}=\left\{b_{1}<\right.$ $\left.\cdots<b_{k+q}<b_{k+q+1}<\cdots<b_{2 k+q} \leq b_{B}\right\}$. We have

$$
b_{k+q+1}+\cdots+b_{2 k+q}<b_{1} \cdots+b_{k}+b_{k+1}+b_{k+q} .
$$

Let $t$ be any integer in $[1, k]$. We have

$$
b_{k+1}+\cdots+b_{k+q}>\left(b_{2 k+q}-b_{1}\right)+\cdots+\left(b_{2 k+q-t+1}-b_{t}\right)+\cdots+\left(b_{k+q+1}-b_{k}\right) .
$$

We clearly have the inequalities

$$
\begin{aligned}
& b_{k+q+1}-b_{k} \geq(q+1) q \\
& b_{k+q+2}-b_{k-1} \geq(q+3) q \\
& \cdots \\
& b_{2 k+q-t-1}-b_{t+2} \geq(q+1+2(k-t-2)) q \\
& b_{2 k+q-t}-b_{t+1} \geq b_{2 k+q-t}-b_{t+1} \\
& b_{2 k+q-t+1}-b_{t} \geq\left(b_{2 k+q-t}-b_{t+1}\right)+2 q \\
& \cdots \\
& \left(b_{2 k+q}-b_{1}\right) \geq\left(b_{2 k+q-t}-b_{t+1}\right)+2 t q
\end{aligned}
$$

We thus obtain

$$
\begin{aligned}
b_{k+1}+\cdots+b_{k+q} & >(t+1)\left(b_{2 k+q-t}-b_{t+1}\right) \\
& +q \sum_{l=0}^{k-t-2}(q+1+2 l)+q \sum_{h=0}^{t} 2 h .
\end{aligned}
$$

Taking into account that $b_{k+q} \leq N-k q$, a dull computation leads to

$$
(t+1)\left(b_{2 k+q-t}-b_{t+1}\right) \leq q\left(N-k^{2}+2 k t+O\left(N^{11 / 12}\right)\right),
$$

when $t=O\left(N^{11 / 24}\right)$. This in turn leads to

$$
b_{2 k+q-t}-b_{t+1} \leq q\left(2 k+O\left(N^{11 / 24}\right)\right),
$$

when $t=\frac{3}{2} N^{11 / 24}+O(1)$.

Let $C$ the cardinality of $\mathcal{C}$. Since $\mathcal{A}=\mathcal{B} \cup \mathcal{C}$, we have

$$
b_{t+1} \leq a_{t+C} \leq a_{A+t+C-2 k-q+1} \leq a_{2 k+q-C-t} \leq b_{2 k+q-t} ;
$$

we choose $u=A+t+C-2 k-q$ and recall that $A-2 k-q \leq C+1=O\left(N^{5 / 12}\right)$, so that Theorem 3 is proven. 
3. We now embark on the proof of Theorem 1 which will follow from Theorem 3 and Proposition 1. Let $\mathcal{A}$ be an admissible subset of $[1, N]$ with maximal cardinality. By [1], we know that $A=2 \sqrt{N}+O\left(N^{5 / 12}\right)$, so we can apply Theorem 3 : there exists integers $u$ and $r$ such that

$$
a_{A-u}-a_{u+1}=q(A-2 u+r),
$$

with $u \in\left[N^{11 / 24}, 2 N^{11 / 24}\right]$ and $r=O\left(N^{11 / 24}\right)$.

We let

$$
\mathcal{D}:=\mathcal{A} \cap\left[a_{u+1}, a_{A-u}\right], \quad t:=A-2 u, \quad \sigma:=[(t-q) / 2],
$$

and we shall apply Proposition 1 with $s=\sigma$ and $s=\sigma+q$ (one readily checks that the conditions of application of Proposition 1 are fulfilled). Let us further denote by $m(s)$ (resp. $M(s))$ the smallest (resp. largest) element in $s^{\wedge} \mathcal{D}$.

As a first step, we show that $a_{1}+a_{2}+\cdots+a_{q}$ cannot be too small. We have

$$
\begin{aligned}
M(\sigma)-m(\sigma) & \geq\left(a_{A-u-\sigma+1}-a_{u+\sigma}\right)+\cdots+\left(a_{A-u}-a_{u+1}\right) \\
& \geq q(2+4+\cdots+2(\sigma-1)=q \sigma(\sigma-1) \\
& =q N+O\left(q N^{23 / 24}\right) .
\end{aligned}
$$

If $\alpha_{q}:=a_{1}+\cdots+a_{q}$ were less than $M(\sigma)-m \sigma-(2 r+1) q$, the intersection of $[m(\sigma), M(\sigma)]$ and $\left[m(\sigma)+\alpha_{q}, M(\sigma)+\alpha_{q}\right]$ would be an interval containing at least $(2 r+1)$ integers in each class modulo $q$. By the property of $\sigma^{\wedge} \mathcal{D}$ established in Proposition 1 , property obviously shared by $\alpha_{q}+\sigma^{\wedge} \mathcal{D}$, the pigeon-hole principle would imply that $\sigma^{\wedge} \mathcal{D}$ and $\alpha_{q}+\sigma^{\wedge} \mathcal{D}$ have an element in common, and this would contradict the admissibility of $\mathcal{A}$. (We may notice that this implies that $a_{1}$ itself is not too small, but we shall not use this fact).

By using the same pigeon-hole argument, we see that the admissibility of $\mathcal{A}$ implies

$$
M(\sigma)+a_{A-u+1}+\cdots+a_{A} \leq m(\sigma+q)+a_{1}+\cdots+a_{u}+(2 r-1) q,
$$

that is to say

$$
a_{A-u-\sigma+1}+\cdots+a_{A-u}+\cdots a_{A} \leq a_{1}+\cdots+a_{u}+a_{u+1}+\cdots+a_{u+\sigma+q}+(2 r-1) q,
$$

whence we deduce

$$
\begin{array}{ll}
\left(a_{A}-a_{1}\right)+\left(a_{A-1}-a_{2}\right) \quad+\cdots+\left(a_{A-u-\sigma+1}-a_{u+\sigma}\right) \leq \\
& a_{u+\sigma+1}+\cdots+a_{u+\sigma+q}+(2 r-1) q .
\end{array}
$$

We have $a_{A-u-\sigma+1}-a_{u+\sigma} \geq q(A-u-\sigma+1-u-\sigma)=q(A-2 u-2 \sigma+1)$ and, by the definition of $\sigma$, we can write

$$
A-2 u-2 \sigma=q+\theta,
$$

where $\theta=0$ if $A-q$ is even and $\theta=1$ if $A-q$ is odd. We thus have

$$
\begin{array}{ll}
u r q+q(1+q+\theta)+q(3+q+\theta) \quad & +\cdots+q(2(u+\sigma)-1+q+\theta) \leq \\
& a_{u+\sigma+1}+\cdots+a_{u+\sigma+q}+(2 r-1) q .
\end{array}
$$


Since $u \geq 2$ and $r \geq 0$, we have

$$
\begin{aligned}
q(u+\sigma)(u+\sigma+q+\theta) \leq & a_{u+\sigma+1}+\cdots+a_{u+\sigma+q}-q \\
\leq & N-(A-u-\sigma-1) q+\cdots+ \\
& N-(A-u-\sigma-q) q-q \\
\leq & N q-A q^{2}+u q^{2}+\sigma q^{2}+\frac{q^{2}(q+1)}{2}-q .
\end{aligned}
$$

We now replace $u+\sigma$ by $\frac{A-q-\theta}{2}$, which leads to

$$
q\left(\frac{A-q-\theta}{2}\right)\left(\frac{A+q+\theta}{2}\right) \leq N q-q^{2}\left(\frac{A+q+\theta}{2}\right)+\frac{q^{2}(q-1)}{2} .
$$

If $A-q$ is even, we get

$$
A^{2}-q^{2} \leq 4 N-2 A q-2 q^{2}+2 q^{2}-2 q,
$$

whence

$$
A^{2}+2 A q+q^{2} \leq 4 N+2 q^{2}-2 q
$$

or

$$
(A+q)^{2} \leq 4 N+2 q^{2}-2 q .
$$

if $q=1$, this is $(A+1)^{2} \leq 4 N$;

if $q \geq 2$, we have

$$
\begin{aligned}
(A+1)^{2} & \leq(A+q)^{2}-(A+q)^{2}+(A+1)^{2} \\
& \leq 4 N+2 q^{2}-2 q-A^{2}-2 A q-q^{2}+A^{2}+2 A+1 \\
\leq & 4 N+2 A(1-q)+(q-1)^{2} \\
\leq & 4 N-(q-1)(2 A-q+1) \leq 4 N .
\end{aligned}
$$

If $A-q$ is odd, we get

$$
A^{2}-(1+q)^{2} \leq 4 N-2 A q-2 q^{2}-2 q+2 q^{2}-2 q .
$$

if $q=1$, this is $A^{2}+2 A+1 \leq 4 N+1$;

if $q \geq 2$, we have

$$
\begin{aligned}
(A+1)^{2} & \leq A^{2}-(1+q)^{2}+2 q+q^{2}+2+2 A \\
& \leq 4 N-2 A(q-1)+q^{2}-2 q+2 \\
& \leq 4 N-(q-1)(2 A-q+1)+1 \\
& \leq 4 N+1 .
\end{aligned}
$$

In all cases, we thus have $(A+1)^{2} \leq 4 N+1$, which ends the proof of our main result. 


\section{References}

[1] Deshouillers J-M. and Freiman G.A., On an additive problem of Erdôs and Straus, 1, Israël J. Math., 92, 1995, 33-43.

[2] Erdôs P., Some remarks on number theory, III. Mat. Lapok 13, 1962, 28-38.

[3] Erdôs P., Nicolas J-L., Sarkőzy A., Sommes de sous-ensembles, Sem. Th. Nb. Bordeaux 3, 1991, 55-72.

[4] Freiman G.A., The addition of finite sets, 1,(Russian), Izv. Vyss. Ucebn. Zaved. Matematika, 6(13), 1959, 202-213.

[5] Straus E.G., On a problem in combinatorial number theory, J. Math. Sci., 1, 1966, 77-80.

J.-M. Deshouillers, Mathématiques Stochastiques, Université Victor Segalen Bordeaux 2, 33076 Bordeaux, France $\bullet E$-mail : j-m.deshouillers@u-bordeaux2.fr

G.A. Freiman, School of Mathematical Sciences, Department of Mathematics, Raymond and Beverly Sackler, Faculty of Exact Sciences, Tel Aviv University, 69978 Tel Aviv, Israel E-mail : grisha@math.tau.ac.il 